\title{
Edge Channels for the Fractional Quantum Hall Effect
}

\author{
C. W. J. Beenakker \\ Philips Research Laboratories, 5600 JA Eindhoven, The Netherlands \\ (Received 11 September 1989)
}

\begin{abstract}
The concept of edge channels is extended from the integer to the fractional quantum Hall effect, and the contribution of an adiabatically transmitted edge channel to the conductance is calculated. The resulting generalized Landauer formula explains the recent observation by Kouwenhoven $e t$ al. of fractional quantization of the Hall conductance at a value unrelated to the bulk properties of the sample.

PACS numbers: $72.20 . \mathrm{My}, 73.40 . \mathrm{Kp}$
\end{abstract}

Can a two-dimensional electron gas with integer filling factor show a fractional quantum Hall effect? The experimental answer is yes, as shown by Kouwenhoven $e t$ $a l{ }^{\prime}$ in a special geometry in which closely spaced current and voltage leads are separated by barriers from the bulk two-dimensional electron gas (2DEG). Quantization of the Hall conductance $G_{H}$ at $\frac{2}{3} \times e^{2} / h$ was measured in this geometry in a $2 D E G$ for which a conventional Hall measurement gave quantization at $1 \times e^{2} / h$. If one would naively apply Laughlin's well-known gauge argument ${ }^{2}$ to this situation, the latter measurement would imply that the quasiparticle excitations in the 2DEG have unit charge $e$, while from the former one would conclude a fractional charge $e^{*}=e / 3$. Note that these are all fourterminal conductance measurements, which a priori one would not have expected to depend on how the current and voltage leads are coupled to the sample.

In the integer quantum Hall effect (IQHE), the concept of edge channels, ${ }^{3}$ in combination with the assumption of adiabatic transport (i.e., absence of inter-edgechannel scattering on short length scales ${ }^{4}$ ), has been successful in explaining the anomalous dependence of $G_{H}$ on the properties of the leads. ${ }^{5-8}$ As shown in Ref. 1, a simple modification of the formula ${ }^{5,6}$ for the anomalies in the IQHE can accurately describe the anomalies in the FQHE as well-suggesting that a generalization of the edge-channel concept to the FQHE should be possible. Edge channels in the IQHE are defined in one-to-one correspondence with the bulk Landau levels: On approaching the boundary of the 2DEG, a Landau level which in the bulk lies below the Fermi level rises in energy because of the presence of the confining potential. The intersection between the $n$th Landau level and the Fermi level defines the location of the $n$th edge channel. This single-electron description is not applicable to the fractional quantum Hall effect (FQHE), which is fundamentally a many-body effect. ${ }^{9}$ Chang and Cunningham $^{10}$ interpreted their earlier experiment on the resistance of a barrier in the FQHE regime in terms of conduction via some form of edge channels, but they did not specify what these channels would be. Indeed, it is not immediately obvious that the concept of independent current channels within the same Landau level has any meaning at all.
In this paper it is shown how the concept of an edge channel can be generalized to the FQHE, and a generalized Landauer formula relating the conductance to the transmission probabilities of the edge channels is derived. In this formula the edge channels contribute with a fractional weight, which is not simply related to any particular quasiparticle charge $e^{*}$. The results obtained explain the experiments of Refs. 1 and 10 , and allow a prediction of the outcome of proposed experiments ${ }^{11}$ to directly measure the quasiparticle charge in the FQHE.

Consider a narrow 2DEG conductor (a "wire") parallel to the $y$ axis, in a perpendicular magnetic field $\mathbf{B}=B \hat{\mathbf{z}}$. In equilibrium, at $T=0$, the electron density $n(r)$ varies as a function of $r \equiv(x, y)$ in such a way that $U-\mu N$ is minimized. Here $\mu$ is the electrochemical potential, $N$ is the total number of electrons, and $U$ is the total energy of the sample (including electrostatic contributions, cf. Ref. 12). A uniform interacting 2DEG of density $n$ in a strong magnetic field has the remarkable property ${ }^{9,12}$ that the internal energy density $u(n)$ has downward cusps at densities $n=v_{p} B e / h$ corresponding to certain fractional filling factors $v_{p}$. As a result, the chemical potential $d u / d n$ has a discontinuity (an energy gap) at $v=v_{p}$, with $d u_{p}^{+} / d n$ and $d u_{p}{ }^{-} / d n$ the two limiting values as $v \rightarrow v_{p}$. At the edges of the wire the density is reduced due to the increase in electrostatic potential energy $\phi(r)$ (which itself is determined both by the external confining potential and by nonuniformities in $n$ ). If $\phi$ varies sufficiently slowly at the edge, one can approximate the internal energy density at $\mathbf{r}$ by the energy density $u(n(\mathrm{r}))$ of the uniform 2DEG with density $n(\mathrm{r})$. The equilibrium electron density is then given by ${ }^{12}$

$$
\begin{aligned}
& n=v_{p} B e / h, \text { if } d u_{p}^{-} / d n<\mu-\phi<d u_{p}^{+} / d n, \\
& d u / d n+\phi(\mathrm{r})=\mu, \text { otherwise. }
\end{aligned}
$$

The resulting stepwise decrease in filling factor $v \equiv n h / e B$ on approaching the edge is illustrated in Fig. 1 . The requirement on the smoothness of $\phi$ for the appearance of a well-defined region at the edge in which $v$ is pinned at the fractional value $v_{p}$ is that the change in $\phi$ within a magnetic length $l_{m} \equiv(h / e B)^{1 / 2}$ is small compared to the energy gap $d u_{p}^{+} / d n-d u_{p}^{-} / d n$. Depending on the smoothness of $\phi$, one thus obtains a series of steps 


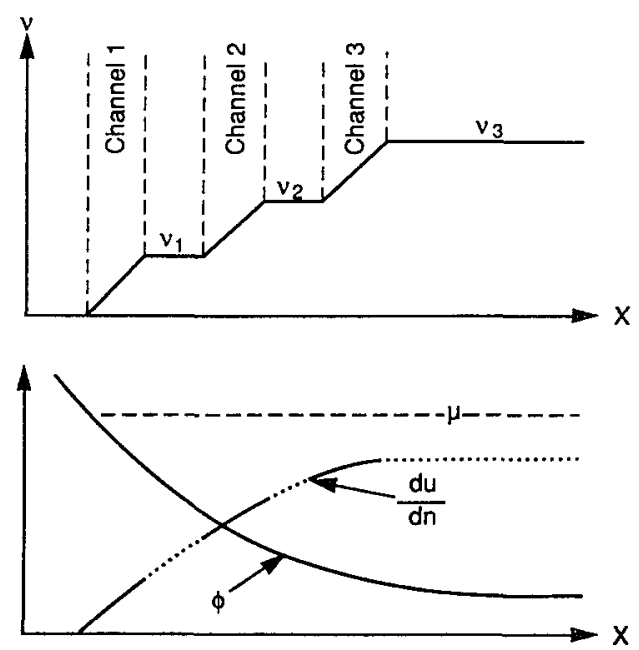

FIG. 1. Schematic drawing of the variation in filling factor $v$, electrostatic potential $\phi$, and chemical potential $d u / d n$, at a smooth boundary in a $2 \mathrm{DEG}$. The dashed line in the bottom panel denotes the constant electrochemical potential $\mu=\phi$ $+d u / d n$. The dotted intervals indicate a discontinuity (energy gap) in $d u / d n$, and correspond in the top panel to regions of constant fractional filling factor $v_{p}$ which spatially separate the edge channels.

at $v=v_{p}(p=1,2, \ldots, P)$, as one moves from the edge towards the bulk. The series terminates in the filling factor $v_{P}=v_{\text {bulk }}$ of the bulk, assuming that in the bulk the chemical potential $\mu-\phi$ lies in an energy gap. The regions of constant $v$ at the edge form bands extending along the wire. These incompressible bands (in which $\delta n / \delta \mu=0$ ) alternate with bands in which $\mu-\phi$ does not lie in an energy gap. The latter compressible bands (in which $\delta n / \delta \mu \neq 0$ ) may be identified as the edge channels of the transport problem, as will now be discussed.

The transport problem is studied, in the spirit of Landauer, ${ }^{13}$ by bringing one end of the conductor in contact with a reservoir at a slightly higher electrochemical potential $\mu+\Delta \mu$, but without changing $\phi$. The resulting change $\Delta n$ in electron density is

$$
\Delta n=\left.\frac{\delta n}{\delta \mu}\right|_{\phi} \Delta \mu=-\left.\frac{\delta n}{\delta \phi}\right|_{\mu} \Delta \mu,
$$

where $\delta$ denotes a functional derivative. In the second equality in Eq. (2) it has been used that $n$ is a functional of $\mu-\phi$, by virtue of Eq. (1). In a strong magnetic field, this excess charge moves along equipotentials with the macroscopic drift velocity $E / B(E \equiv \partial \phi / e \partial \mathrm{r}$ being the electrostatic field). The component $v_{\text {drift }}$ of the drift velocity in the $y$ direction (along the wire) is

$$
v^{\prime} \text { drift }=\hat{\mathbf{y}} \cdot\left(\mathrm{E} \times \mathbf{B} / B^{2}\right)=-(1 / e B) \partial \phi / \partial x \text {. }
$$

The nonequilibrium current density $j=-e \Delta n v^{\prime}$ drift becomes simply

$$
j=-\frac{e}{h} \Delta \mu \frac{\partial v}{\partial x}
$$

(a)



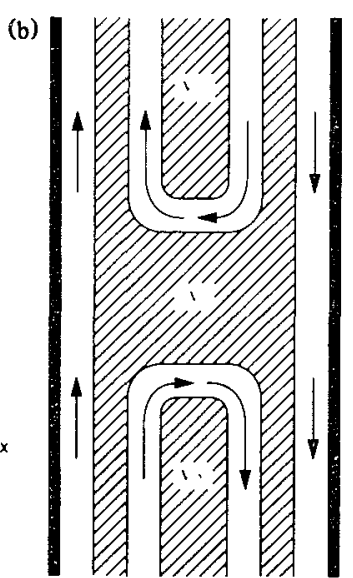

FIG. 2. Schematic drawing of the incompressible bands (hatched) of fractional filling factor $v_{p}$, alternating with the edge channels (arrows indicate the direction of electron motion in each channel). (a) A uniform conductor; (b) a conductor containing a barrier of reduced filling factor (as in the experiment of Ref. 10).

It follows from Eq. (4) that the incompressible bands of constant $v=v_{p}$ do not contribute to $j$. The reservoir injects the current into the compressible bands at one edge of the conductor only (for which the sign of $\partial v / \partial x$ is such that $j$ moves away from the reservoir). I define the generalized concept of the edge channel with index $p=1,2, \ldots, P$ as that compressible band which is flanked by incompressible bands at filling factors $v_{p}$ and $v_{p-1}$. (The outermost band from the center of the conductor, which is the $p=1$ edge channel, is included by defining formally $v_{0} \equiv 0$.) The arrangement of alternating edge channels and incompressible bands is illustrated in Fig. 2(a). Note that different edges may have a different series of edge channels at the same magnetic field value, depending on the smoothness of the potential $\phi$ at the edge (which, as discussed above, determines the incompressible bands that exist at the edge). This is in contrast to the situation in the IQHE, where a one-toone correspondence exists between edge channels and bulk Landau levels. ${ }^{3}$ In the FQHE an infinite hierarchy of energy gaps exists, in principle, corresponding to an infinite number of possible edge channels- of which only a small number (corresponding to the largest energy gaps) will be realized in practice.

The current $I_{p}=(e / h) \Delta \mu\left(v_{p}-v_{p-1}\right)$ injected into edge channel $p$ by the reservoir follows directly from Eq. (4), on integration over $x$. The total current $I$ through the wire is $I=\sum_{p}^{P}-1 I_{p} T_{p}$, if a fraction $T_{p}$ of the injected current $I_{p}$ is transmitted to the reservoir at the other end of the wire (the remainder returning via the opposite edge). For the conductance $G \equiv e I / \Delta \mu$ one thus obtains the generalized Landauer formula for a two-terminal conductor

$$
g \equiv \frac{h}{e^{2}} G=\sum_{p=1}^{P} T_{p} \Delta v_{p},
$$


which differs from the usual Landauer formula by the presence of the weight factors $\Delta v_{p} \equiv v_{p}-v_{p}-1$. In the IQHE, $\Delta v_{p}=1$ for all $p$ so that the familiar formula with unit weight factor ${ }^{3,5}$ is recovered. Note that if $T_{p}=1$ for all $p$, then Eq. (5) reduces to $g=v_{P}$ which is the accepted expression for the quantized two-terminal conductance in the FQHE.

A multiterminal generalization of Eq. (5) for a twoterminal conductor is easily constructed, following Büttiker: ${ }^{14}$

$$
\begin{aligned}
& I_{a}=\frac{e}{h} v_{\alpha} \mu_{\alpha}-\frac{e}{h} \sum_{\beta} T_{\alpha \beta} \mu_{\beta}, \\
& T_{\alpha \beta}=\sum_{p=1}^{P_{\beta}} T_{p, \alpha \beta} \Delta v_{p} .
\end{aligned}
$$

Here $I_{\alpha}$ is the current in lead $\alpha$, connected to a reservoir at electrochemical potential $\mu_{a}$, and with fractional filling factor $v_{a}$. Equation (6b) defines the transmission probability $T_{\alpha \beta}$ from reservoir $\beta$ to reservoir $\alpha$ (or the reflection probability, for $\alpha=\beta$ ), in terms of a sum over the generalized edge channels in lead $\beta$. The contribution from each edge channel $p=1,2, \ldots, P_{\beta}$ contains the weight factor $\Delta v_{p} \equiv v_{p}-v_{p-1}$, and the fraction $T_{p, \alpha \beta}$ of the current injected by reservoir $\beta$ into the $p$ th edge channel of lead $\beta$ which reaches reservoir $\alpha$. Apart from the fractional weight factors, the structure of Eq. (6) is the same as that of the usual Büttiker formula. ${ }^{14}$ Note that (in contrast to the anticipation in Ref. 10) the weight factors $\Delta v_{p}$ are not in general given by $e^{*} / e$ (with $e^{*} \equiv e / q$ the charge of the quasiparticle excitations in a lead at fractional filling factor $p / q$ ). The physical origin for the absence of a one-to-one correspondence between the edge-channel weight and the charge of the excitations of the incompressible FQHE state is that the edge channels themselves are not incompressible. I will return to this important point at the end of this paper.

In the experiment of Chang and Cunningham, ${ }^{10} \mathrm{a}$ negatively biased gate is placed across a segment of a narrow $2 D E G$, which has the effect of locally reducing the filling factor. Consider the case that the chemical potential lies in an energy gap at $v=v_{P}$ in the part of the 2DEG not covered by the gate, and at $v^{\prime}=v_{P^{\prime}}<v_{P}$ underneath the gate. The arrangement of edge channels and incompressible bands is illustrated in Fig. 2(b). It is assumed for simplicity that the potential barrier created by the gate is sufficiently smooth that scattering between the edge channels can be neglected. All transmission probabilities in this regime of adiabatic transport are either 0 or 1: $T_{p}=1$ for $1 \leq p \leq P^{\prime}$, and $T_{p}=0$ for $P^{\prime}<p \leq P$. Equation (5) then tells us that $g=v_{P^{\prime}}$, as found experimentally. ${ }^{10}$

In the experiment of Kouwenhoven et al.,' a fourterminal measurement of the Hall conductance $G_{H} \equiv\left(e^{2}\right)$ $h) g_{H}$ in the FQHE regime is made, in a geometry shown schematically in Fig. 3(a). One current lead and one voltage lead contain a barrier. These two leads are adja-

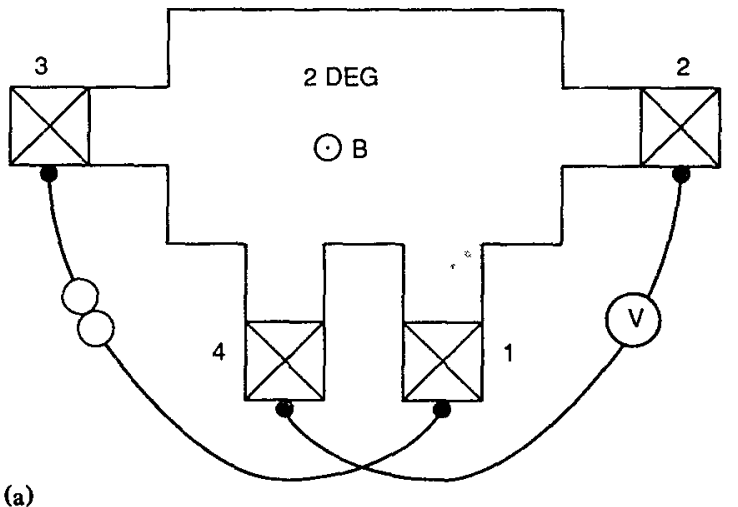

(a)

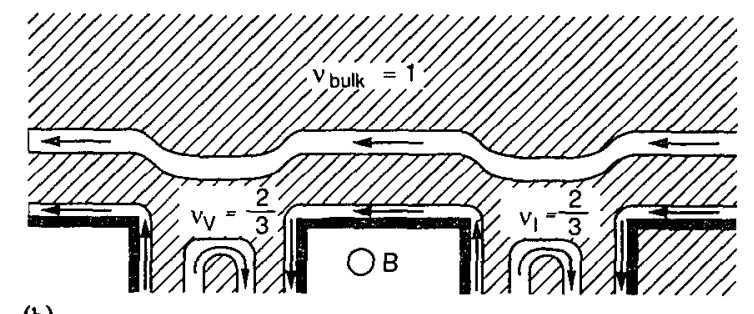

(b)

FIG. 3. (a) Schematic drawing of the experimental geometry of Ref. 1. The crossed squares are contacts to the 2DEG. One current lead and one voltage lead contain a barrier (shaded), of which the height can be adjusted by means of a gate (not drawn). The current flows between contacts 1 and 3 , the voltage is measured between contacts 2 and 4 . (b) Arrangement of incompressible bands (hatched) and edge channels near the two barriers, for the case studied in Ref. 1 of $v_{\text {bulk }}=1, v_{I}=v_{V}=\frac{2}{3}$.

cent at the edge of the 2DEG, and separated by a small distance of $2 \mu \mathrm{m}$. Figure $3(\mathrm{~b})$ illustrates the arrangement of edge channels and incompressible bands for the case that the chemical potential lies in an energy gap for the bulk 2DEG (at $v=v_{\text {bulk }}$ ), as well as for the two barriers (at $v_{l}$ and $v_{V}$ for the barrier in the current and voltage leads, respectively). Adiabatic transport is assumed over the barrier, as well as from barrier $I$ to barrier $V$ (for the magnetic field direction indicated in Fig. 3). The Büttiker equations for this geometry in the IQHE regime have the solution ${ }^{6} g_{H}=\max \left(N_{I}, N_{V}\right)$, with $N_{I}$ and $N_{V}$ the number of spin-split Landau levels occupied at barriers $I$ and $V$. Equation (6a) for the FQHE has the same structure, and thus the same solution, after the substitution $N_{I, V} \rightarrow v_{I, V}$. The Hall conductance is therefore given by

$$
g_{H}=\max \left(v_{I}, v_{V}\right) \leq v_{\text {bulk }},
$$

so that the quantized Hall plateaus are determined by the fractional filling factors of the current and voltage leads, not of the bulk 2DEG. A more general formula for $g_{H}$ valid also in between the quantized plateaus is given in Ref. 1, and is shown there to be in quantitative 
agreement with the experiment.

The concept of generalized edge channels for the FQHE introduced here is expected to open up a new class of transport experiments, by analogy with the experiments on edge channels in the IQHE regime. It is emphasized that the transmission probabilities of the generalized edge channels in Eqs. (5) and (6) refer to quasiparticle excitations of the "normal" compressible bands in the $2 \mathrm{DEG}$, corresponding to regions without an excitation gap, and not to the quasiparticle excitations of charge $e^{*}$ of the incompressible bands. For this reason one would expect (even in the absence of an explicit calculation of the transmission probabilities) that an Aharonov-Bohm experiment in the FQHE regime would have the $h / e$ and not the $h / e^{*}$ periodicity, or more generally that proposed transport experiments " to directly measure the charge of the quasiparticles in the FQHE would measure the normal electron charge $e$ rather than the fractional charge $e^{*}$

I thank L. P. Kouwenhoven, B. J. van Wees, and C. J. P. M. Harmans for communicating their results to me at an early stage. I also thank $H$. van Houten, D. van der Marel, and M. F. H. Schuurmans for stimulating discussions, and A. M. Chang for a preprint of Ref. 10.
${ }^{1}$ L. P. Kouwenhoven, B. J. van Wees, N. C. van der Vaart, C. J. P. M. Harmans, C. E. Timmering, and C. T. Foxon (to be published).

${ }^{2}$ R. B. Laughlin, Phys. Rev. B 23, 5632 (1981).

${ }^{3}$ B. I. Halperin, Phys. Rev. B 25, 2185 (1982); P. Streda, J. Kucera, and A. H. MacDonald, Phys. Rev. Lett. 59, 1973 (1987); J. K. Jain and S. A. Kivelson, Phys. Rev. B 37, 4276 (1988).

${ }^{4}$ L. I. Glazman and M. Jonson, J. Phys. Condens. Matter 1, 5547 (1989); T. Martin and S. Feng (to be published).

${ }^{5}$ M. Büttiker, Phys. Rev. B 38, 9375 (1988).

${ }^{6}$ B. J. van Wees, E. M. M. Willems, C. J. P. M. Harmans, C. W. J. Beenakker, H. van Houten, J. G. Williamson, C. T. Foxon, and J. J. Harris, Phys. Rev. Lett. 62, 1181 (1989).

${ }^{7}$ S. Komiyama, H. Hirai, S. Sasa, and S. Hiyamizu, Phys. Rev. B 40, 12566 (1989).

${ }^{8}$ B. W. Alphenaar, P. L. McEuen, R. G. Wheeler, and R. N. Sacks (to be published).

${ }^{9}$ R. B. Laughlin, Phys. Rev. Lett. 50, 1395 (1983).

${ }^{10}$ A. M. Chang and J. E. Cunningham (to be published).

"S. A. Kivelson and V. L. Pokrovsky, Phys. Rev. B 40, 1373 (1989).

12B. I. Halperin, Helv. Phys. Acta 56, 75 (1983).

${ }^{13}$ R. Landauer, IBM J. Res. Dev. 32, 306 (1988).

${ }^{14}$ M. Büttiker, Phys. Rev. Lett. 57, 1761 (1986). 